Voix et Images

voixetimages

\title{
Suzanne Jacob, à l'ombre des jeunes femmes en fuite
}

\section{Lori Saint-Martin}

Volume 21, numéro 2 (62), hiver 1996

Suzanne Jacob

URI : https://id.erudit.org/iderudit/201236ar

DOI : https://doi.org/10.7202/201236ar

Aller au sommaire du numéro

\section{Éditeur(s)}

Université du Québec à Montréal

\section{ISSN}

0318-9201 (imprimé)

1705-933X (numérique)

Découvrir la revue

\section{Citer cet article}

Saint-Martin, L. (1996). Suzanne Jacob, à l'ombre des jeunes femmes en fuite. Voix et Images, 21(2), 250-257. https://doi.org/10.7202/201236ar
Résumé de l'article

Résumé

Au point de départ du présent article se trouve la lecture qu'effectue Alice fardine de la femme et de la modernité dans la théorie française contemporaine. Dans les oeuvres en prose de Suzanne Jacob, le féminin est précisément ce qui échappe à la maîtrise, ce qu 'on ne peut saisir, immobiliser. Les protagonistes multiplient les départs, les amours, toutes formes de résistance devant l'écrasante uniformité de la vie en société. d'utilisation que vous pouvez consulter en ligne.

https://apropos.erudit.org/fr/usagers/politique-dutilisation/ 


\title{
Suzanne Jacob, à l'ombre des jeunes femmes en fuite
}

\author{
Lori Saint-Martin, Université du Québec à Montréal
}

\begin{abstract}
Au point de départ du présent article se trouve la lecture qu'effectue Alice Jardine de la femme et de la modernité dans la théorie française contemporaine. Dans les ceuvres en prose de Suzanne Jacob, le féminin est précisément ce qui échappe à la maîtrise, ce qu'on ne peut saisir, immobiliser. Les protagonistes multiplient les départs, les amours, toutes formes de résistance devant l'écrasante uniformité de la vie en société.
\end{abstract}

Toute l'œuvre de Suzanne Jacob, jusqu'à L'Obéissance, qui marque un nouveau départ, met en scène - et en jeu - la même femme, la trentaine à peine entamée, grande voyageuse, amoureuse volage, fuyante et multiple. Flore Cocon, Laura Laur, La Passion selon Galatée, Maude, Les Aventures de Pomme Douly: les titres dessinent déjà un parcours. Si le nom propre au féminin revient de manière quasi systématique, s'il s'agit de noms parfaitement ludiques, invraisemblables, en un mot écrits, c'est, on le voit bien, parce que l'œuvre ne fait que déployer, à l'infini, les avatars démultipliés d'une femme toujours pareille à elle-même et toujours inattendue. Mieux, je dirai que, au-delà de l'anecdote, ce qui nous est donné à lire chez Suzanne Jacob est une mise en texte infinie de la figure du féminin. Non pas de la femme moderne (ces femmes sont tout sauf typiques), ni de la féminité stéréotypée (chacune s'enorgueillit à sa manière d'être "la plus toffe, la plus gars, la plus sans cœur ${ }^{1}$ "), mais de la féminité au sens d'une certaine théorie française moderne: ce qui échappe à la maîtrise. Ou encore, comme le dit Alice Jardine, ce qu'il a fallu reconceptualiser de "non-savoir" dans les grands métarécits qu'a remis en cause la crise de la légitimation:

Cet autre-qu'eux est presque toujours un genre "d'espace " (que la narration ne contrôle plus) et c'est un espace qui a été codé de féminin, de femme [...]

1. La Survie, Montréal, Le Biocreux, 1980, p. 25. Désigné par $S$ dans le texte, suivi du folio. 
L'objet produit par ce processus n'est ni une personne, ni une chose, mais un horizon vers lequel tend le processus: une gynéma. Cette gynéma est un effet de lecture, une femme-en-effet qui n'est jamais stable et ne possède aucune identité ${ }^{2}$.

Évidemment - et c'est le propos de Jardine - il s'agit là d'un féminin fantasmatique, détaché des femmes réelles et à plus forte raison du féminisme. Point n'est besoin des femmes pour qu'advienne cette féminité-là, pur signifiant ${ }^{3}$, "espace" dépersonnalisé que le maître théoricien manipule à son gré. En revanche, lorsque se croisent féminin au sens de la théorie française et expérience vécue des femmes, comme c'est le cas chez Suzanne Jacob, on obtient des effets saisissants. Nous est donné à découvrir, dans son œuvre, le féminin dans sa version métaféministe ${ }^{4}$ et postmoderne ${ }^{5}$. Le féminin de jeunes femmes modernes, féministes ou du moins femmes libres, pleinement contemporaines, qui vont et viennent, multipliant les amants et les évasions.

La femme jacobienne est donc une femme "réelle" au sens textuel mais aussi, simultanément, une femme-en-effet, une femme qui n'appartient à personne et qui possède - ou dépossède - de multiples interprétations :

Flore Cocon passe pour bien des choses. Certains la croient nymphomane. On la dit hystérique. Aux yeux des uns, c'est une putain éduquée. Pour les autres, c'est une marginale éblouissante au bouillonnement cérébral tumultueux ${ }^{6}$.

2. Alice Jardine, Gynésis. Configurations de la femme et de la modernité, traduit de l'américain par Patricia Baudoin, Paris, Presses universitaires de France, 1991, p. 24.

3. Nancy $K$. Miller rappelle que "the "end of man" in no way precludes the reinscription of woman as Other " ("la "fin de l'Homme" n'empêche nullement la réinscription de la femme comme Autre") et que seuls ceux qui jouissent du prestige découlant du statut d'Homme et d'Auteur peuvent jouer à le remettre en cause. Voir "The Text's Heroine: A Feminist Critic and Her Fictions", Subject to Change: Reading Feminist Writing, New York, Columbia University Press, 1988, p. 70.

4. Dans les textes que j'appelle métaféministes, et qui sont légion depuis le milieu des années quatre-vingt, un retour au narratif s'accompagne d'un discours qui intègre nombre d'acquis du féminisme - ici, notamment, la liberté sexuelle et professionnelle et la mobilité - qui vont désormais de soi au lieu de faire l'objet d'une revendication. Il s'agit non pas d'un rejet du féminisme, mais d'une intégration de ses prémisses, dont on n'a plus besoin de se réclamer explicitement. Voir "Le métaféminisme et la nouvelle prose féminine au Québec ", Voix et Images, vol. XVIII, no 1 (52), automne 1992, p. 7888, repris dans Lori Saint-Martin (dir.), L'Autre Lecture. La critique au féminin et les textes québécois, Montréal, XYZ éditeur, 1993, p. 161-170.

5. Rappelons que la question des rapports entre féminisme et postmodernisme demeure ouverte, certaines féministes jugeant que la remise en cause des identités stables (des grands métarécits, selon le terme de Lyotard) qui est à la base du postmodernisme "dé-légitime" aussi la construction sociale de la féminité, d'autres affirmant que cette réflexion déstabilisante prive les femmes, trop tôt, de ce qu'elles n'ont jamais possédé : un statut de sujet souverain en même temps qu'une conscience de groupe opprimé. L'ouvrage collectif de Linda Nicholson, Feminism/Postmodernism (Londres, Routledge, 1990), offre une utile synthèse de divers points de vue sur le sujet.

6. Suzanne Jacob, Flore Cocon, Montréal, Parti pris, 1978, p. 12. Désigné par FC dans le texte, suivi du folio. 
Sur la femme jacobienne, personne n'a le dernier mot, surtout pas l'instance narrative. Elle étonne, bouleverse, fait vaciller les certitudes - "On ne sait jamais rien quand Laura est là ${ }^{7}$ " - et s'échappe de tous les cadres. Aérienne, fugueuse, sujette à des intermittences, elle cristallise tous les désirs, déstabilise tous les savoirs. C'est en ce sens précis qu'elle rejoint l'excès, le débordement, qui marquent le féminin dans la théorie française ${ }^{8}$.

Laura Laur n'a rien de mystérieux, affirme Louise Milot, elle n'existe tout simplement pas; c'est un personnage "laissé en creux, sorte de négatif ${ }^{9}$ ". À la lumière des œuvres parues depuis, on voit qu'il s'agit là non pas d'une faiblesse de composition, mais bien d'une stratégie d'écriture. "La/une femme jamais ne se re(n)ferme en un volume", affirme Luce Irigaray ${ }^{10}$. Pas de nature féminine donc, ni même de caractère arrêté, mais d'innombrables figures du féminin:

Il n'y a pas une nature de Galatée, se dit-elle, il y a diverses architectures de Galatée, suivant les villes et les circonstances ${ }^{11}$.

Pomme avait plusieurs côtés d'elle-même parmi lesquels figuraient un côté maternel et un côté apitoyé ${ }^{12}$.

Multiple, on ne le demeure qu'au prix de fuites stratégiques. Partir toujours, revenir parfois: "Maude ne respecte aucune entente [...] Elle s'est arrogé le droit de disparaître ${ }^{13}$. "Mieux que sa présence, ses éclipses définissent la femme jacobienne, la font exister. Voyageuse sans bagages, elle affectionne les taxis, les avions, tous les lieux qui portent en eux l'annonce et la promesse du départ: "Je passerais bien la nuit ici à regarder les feux se balader sur la piste." ( $S$, p. 103) Pomme Douly vit pour ressentir "la joie furieuse qui s'empare d'elle lorsqu'elle regarde sa valise s'éloigner sur un convoyeur d'aéroport " $(A P D, 46)$.

Ne rien posséder, sinon l'espace d'un instant. Le dépouillement rend ces femmes heureuses, légères. Elles ne peuvent travailler qu'à la pige, et encore, si peu, si peu. Acheter ou voler, peu importe - Laura Laur vole les parfums coûteux que son amant rêve de lui offrir, comme pour affirmer qu'elle n'a nul besoin de lui - , ou encore jouir de l'objet par le seul regard. Un personnage de La Survie achète, pour le plaisir, "du papier à

7. Id., Laura Laur, Paris, Seuil, 1983, p. 143. Désigné par $L L$ dans le texte, suivi du folio.

8. Voir, entre beaucoup d'autres, Jacques Lacan, Encore, Paris, Seuil, 1975, p. 34: «[I]l y a toujours quelque chose qui chez elle échappe au discours."

9. Louise Milot, "Comment nommer sans dire ", Lettres québécoises, n ${ }^{\circ} 32$, hiver 1983-1984, p. 23.

10. Luce Irigaray, Speculum. De l'autre femme, Paris, Minuit, 1974, p. 4 de couverture.

11. Suzanne Jacob, La Passion selon Galatée, Paris, Seuil, 1987, p. 235. Désigné par PG dans le texte, suivi du folio.

12. Id., Les Aventures de Pomme Douly, Montréal, Boréal, 1988, p. 12. Désigné par APD dans le texte, suivi du folio.

13. Id., Maude, Montréal, NBJ, 1988, p. 42. Désigné par $M$ dans le texte, suivi du folio. 
lettres, un cahier à dessins, des crayons feutre, de l'encre de Chine, des cassettes vierges, un foulard de coton rouge, un petit pot d'olives noires, un bracelet de marbre" ( $S$, p. 38). Bref, elle s'offre l'écriture, la musique et le plaisir (couleurs, gourmandise, parures). Puis elle marche dans la ville, sans but, et abandonne le sac qui commençait à lui peser. Un homme qui la suivait tombe amoureux de ce geste léger, libre, souverain.

L'espace privilégié de cette femme est l'hôtel, figure à la fois de l'accueil et de l'anonymat: les chambres où elle fait l'amour avec un presque inconnu rencontré dans un bar ou un aéroport, les halls où elle fait oublier aux hommes leurs rendez-vous d'affaires. Et, aussitôt après la chambre, la rue qui l'efface: "Je n'avais pas fait dix pas sur le trottoir que j'avais tout oublié." ( $P G$, p. 161) Elle aime encore les salles de cinéma où on se rend en taxi, seule; peu importe le film à condition de pouvoir se noyer dans le rapide flot des images. À croire que l'ultime victoire consiste à disparaître vraiment. Jamais il ne s'agit de suicide, mais bien d'une évasion réussie:

Il est si tard cependant et voilà je lui glisse des mains malgré moi. Le soleil va se lever, une petite brume de rien du tout s'agite sur le canal ou c'est une vision de moi m'évaporant. Ce n'est pas grave, ça ne fait pas mal. C'est bon. Ça fait du bien. ( $S$, p. 99)

On était très lisse, presque rien, c'était libre. ( $M$, p. 10)

Elle peut s'abandonner à la distraction, lui accorder toute son attention. ( $M$, p. 71)

Attardons-nous un instant à l'exemple de Laura Laur, personnageénigme ${ }^{14}$ qui déjoue toutes les attentes. En son nom, la double initiale L-L évoque fatalement le pronom féminin (elle-elle), fait sonner le féminin à l'état presque pur. Comme la protagoniste nous est donnée à voir à travers le récit de quatre hommes (deux amants, deux de ses frères), une lecture hâtive pourrait faire croire à une forme d'aliénation discursive. Privée de parole propre, ni narratrice ni focalisatrice, Laura Laur n'existerait selon cette lecture que dans le regard des hommes qui l'entourent ${ }^{15}$. Elle se réduirait, selon l'expression de Jeanne Lapointe décrivant la femme dans le roman masculin traditionnel, à "l'objet de la quête, à la fois poursuivi et redouté ${ }^{16}{ }_{n}$. Et pourtant, rien n'est aussi simple avec Laura Laur. Est bien pris, ici, qui croyait prendre. Le silence de Laura Laur la grandit,

14. La publicité qui a lancé le livre posait avec insistance la question: "Qui est Laura Laur?», emblématique et toujours ouverte.

15. Voir Louise Milot, loc. cit., p. 23. Christiane Lahaie affirme au contraire que le silence du personnage serait plutôt "une forme de discipline que Laura se donnerait à ellemême, à savoir, à quoi bon s'exprimer quand personne n'écoute", si bien qu'elle demeure insaisissable. Voir "Alice s'en va au cinéma, ou comment museler le roman féministe ", Recherches féministes, vol. VII, ${ }^{\circ}$ 2, 1994, p. 83.

16. Jeanne Lapointe, "Perspectives féministes en littérature", Roberta Mura (dir.), Un savoir à notre image? Critiques féministes des disciplines, Montréal, Adage, 1991, p. 44. 
si bien qu'elle devient l'unique référent, voire l'unique énonciatrice, des discours que déploie le roman. Elle est "le nom qui hante" et qu'on ne peut s'empêcher de prononcer ( $L L$, p. 169). Là où les hommes croient parler, c'est elle qui s'exprime par leur bouche: "tout ce que je dis vient de Laur" ( $L L$, p. 9), affirme son jeune frère, alors que son amant, Gilles, voit le monde à travers.l'écran des mots de Laura: "Les visages sont hermétiquement fermés dans les voitures, elle a raison, des huîtres." ( $L L$, p. 46) Même le frère aîné qui prétend la détester épouse son discours: "[T]out ce questionnement vient de Laura, lui appartient. " $L L$, p. 166) Malgré son apparente absence, la voix de Laura Laur est donc l'unique voix narrative du roman, dur diamant se fractionnant en reflets multiples, la voix même, pourrait-on dire, de l'énonciation au féminin: "Elle a une force incomparable dans le ton, même si elle murmure." ( $L L$, p. 35) Comment ne pas penser à cet in-fini du discours au féminin qu'évoque Luce Irigaray: simultanéité, fluidité, capacité de se "re-toucher" à l'infini sans jamais se "constituer en quelque unité ${ }^{17}$ "? Laura Laur ou l'énonciation au féminin à l'état pur, sans énoncé, un discours qui s'annule au moment même où il s'énonce, pour que rien, jamais, ne soit définitif: "[T]out s'est perdu au fur et à mesure qu'il neigeait dans la neige, et rien ne s'est inscrit. " ( $L L$, p. 39) Loin de se laisser réduire au statut d'objet muet du discours masculin, Laura en est le sujet paradoxal au moment précis où elle s'amuse à le détruire. Car rien ne se dit sans elle.

Laura Laur, c'est encore celle qui n'a ni projets ni domicile fixe, qui ne possède rien, pas même les vêtements qu'elle porte, empruntés çà et là, celle en somme qui n' "offrait pas de prises" ( $L L$, p. 78$)$. Elle vit dans l'entre-deux, les portes béantes, le manteau déboutonné, les cheveux au vent. Est-elle blonde ou brune? De quelle couleur sont ses yeux? On n'arrive jamais à le savoir. Qui la contemple demeure ainsi fidèle à son enseignement, elle qui "était contre savoir une chose" ( $L L$, p. 13). Jusqu'à son nom emblématique qu'elle met en jeu, donnant pour toute réponse à la question "Comment tu t'appelles?" un suave "Ça dépend" ( $L L$, p. 111$)^{18}$.

Inutile, comprend enfin Gilles, de vouloir la saisir: "Il pouvait casser l'écran, il ne trouverait rien. Il pouvait casser Laura, il ne trouverait rien." ( $L L$, p. 86) Ce qui ne signifie pas pour autant qu'elle soit vide. Si les images ne sont pas "dans" le téléviseur, elles n'en existent pas moins par lui seul. Écran sur lequel se profilent les images les plus variées, Laura Laur résiste à toute tentative de démontage comme à tout diagnostic hystérie, dépression? - qui aurait pour effet de l'enfermer dans une essence. Image saisissante : abandonné par elle, Gilles s'enferme pendant des semaines dans une chambre de motel avec sa photo, qu'il laisse sur

17. Luce Irigaray, Ce sexe qui n'en est pas un, Paris, Minuit, 1977, p. 76.

18. Voir aussi $S$, p. 57: "Je m'appelle Frédérique, mais ce n'est pas si simple." 
place au moment de réintégrer la vie conjugale. La femme de ménage la ramasse et l'oublie dans la poche de son tablier, qu'elle met au lavage ( $L L$, p. 133). Laura Laur ou l'image mobile, l'irreprésentable, l'impossible à figer.

C'est dans cette optique qu'il faut relire la chute du roman: le présumé suicide d'une Laura aliénée et masochiste au moment où son frère aîné, sa belle-sœur, Gilles et sa femme attendent anxieusement son apparition. Cette mort devient alors un refus de comparaître ultime, irrévérencieux et, pour tout dire, joyeux. On la croyait captive, derrière la porte fermée; erreur, elle était déjà ailleurs. Tout comme Flore Cocon qui traverse le cimetière de la Côte-des-Neiges et sort, indemne, par l'autre bout ( FC, p. 121).

Traverser la vie et la mort, d'un seul mouvement fluide. Traverser aussi la frontière entre les sexes, car à tout moment, les catégories prétendument figées du féminin et du masculin sont remises en cause. Oui, la femme est celle qui, de manière toute classique, doute de sa beauté ${ }^{19}$ ou encore s'effondre dans la rue et doit être sauvée par un inconnu; seulement, elle fait alors appel non pas à la force de l'homme mais à son infinie tendresse, à sa capacité de s'oublier, bref à ce que le texte décrit comme sa part féminine. Mais la femme jacobienne est surtout celle qui séduit, celle qui prend. Au lit, elle déroute son amant en s'appropriant le rôle actif:

Se soumettre aux caresses, à la bouche, aux doigts brûlants qui s'attardent à ses fentes à lui, à ses trous. Non! Il n'est pas comme ça. Il n'est pas une femme après tout. Il voudrait. Geindre, gémir, se tordre, supplier, s'offrir, comme les femmes dans les films... ( $L L$, p. 73$)$

Même jeu ailleurs: "Babey dit que je ne suis pas une femme", dit Galatée ( $P G$, p. 117); "Flore lui disait qu'il était une femme." ( $F C$, p. 117) En plus d'évoquer la double féminité, comme je l'ai laissé entendre plus haut, le nom de Laura Laur pourrait se lire comme une mise en texte de l'androgynie: forme féminine (en "a") et masculine d'un même nom. Gilles achète, pour l'offrir à Laura Laur, un curieux cendrier, formé des os de la "main droite d'un humain mâle ou femelle" ( $L L$, p. 94); c'est l'incertitude quant au sexe qui rend l'objet attirant. Même hésitation pour Flore Cocon: "Flore Cocon, au répertoire des êtres vivants, figure sous le mot femme. Sous le mot homme. Sous le mot humain." (FC, p. 72) Le refus des catégories de sexe correspond non pas à une mise entre parenthèses du désir, mais au contraire à un appel à toutes les fêtes des sens. Partout l'auteure sème le gender trouble, pour reprendre le terme de Judith Butler, brouille la rassurante frontière qui sépare le masculin du féminin.

19. "Je suis belle? C'est vous qui le dites. Pour le moment, moi je ne le sens pas." (FC, p. 76) 
Tout comme s'abolit la barrière entre nature et artifice lorsqu'on dit à Gala: "Tu es beaucoup plus naturelle avec le maquillage." ( $P G$, p. 214)

Lorsque Flore Cocon s'écrie: "J'ai du ressort. Je suis jeune. Je suis bandée. Je suis bandée " $(F C$, p. 77$)$, elle donne sérieusement à réfléchir. Il faut bien se garder de voir là une quelconque référence à une castration féminine; l'auteure connaît sa psychanalyse ${ }^{20}$ et n'y croit guère, sinon par jeu. Avec la femme jacobienne, on s'approche peut-être plutôt de cette bisexualité fondamentale que Freud croyait propre à tous les êtres humains, et que Luce Irigaray entre autres dit particulièrement féminine du fait que le premier objet d'amour-passion de la petite fille est une autre femme, sa mère ${ }^{21}$ : "Pomme aimait désespérément les hommes depuis l'âge de onze ans [...] Pomme, il faut dire, aimait désespérément les femmes depuis l'âge de quatorze ans." ( $A P D$, p. 31) La bisexualité est, elle aussi, signe d'un refus des frontières: il n'y a ni hommes ni femmes, seulement des corps désirants, des énergies qui circulent.

Certes, le corps féminin sexué mobilise quelques affects négatifs: Flore ne voulait pas être menstruée, Laura Laur a une relation plus qu'orageuse avec sa mère et avec sa propre maternité ${ }^{22}$. Reste que le désir seul s'oppose à la réglementation sociale qui marque, selon Jacob, chaque instant de notre vie. L'image des conducteurs immobilisés dans leur voiture à l'heure de pointe, récurrente, est l'emblème d'un conformisme galopant et d'une surconsommation qu'on confond bien à tort avec la liberté. Sur le plan discursif, le cliché traduit la même soumission aveugle à l'autorité, d'où le besoin de le discréditer en le mettant inlassablement en scène: "C'est beau, un pupitre en ordre parce que les yeux sont le miroir de l'âme et parce que ce qui se conçoit bien s'annonce clairement." ( $S$, p. 23) Entre La Survie et La Passion selon Galatée, la part accordée au cliché s'amenuise au profit d'une écriture plus déliée, marquée par l'effacement progressif de l'enchaînement logique de même que par les dérapages sonores ou sémantiques ${ }^{23}$, pendant formel de la force transgressive qui anime les personnages, et dont la passion est l'une des formes privilégiées. En effet, à multiplier les coups de foudre pour les êtres et les choses, on évite la glu de la stabilité amoureuse, qui terrorise tant de personnages. Seul un amour peut guérir d'un autre amour, c'est dans l'ordre, voulu, des choses. "Il ne va jamais revenir, dit Pomme, et il en viendra un

20. En témoigne entre autres une paraphrase ironique de Lacan: "Personne ne peut dire d'une femme que c'est quelqu'un." ( $P G$, p. 67)

21. Luce Irigaray, Le Corps-à-corps avec la mère, Montréal, Pleine Lune, 1981, p. 31.

22. Selon Jean Anderson, l'identité instable de personnages comme Laura ou Flore est liée au rapport à la famille et, plus particulièrement, à la mère. Voir "Mother, Mirror, Self: The "New" Writing of Suzanne Jacob", New Zealand Journal of French Studies, vol. IX, $\mathrm{n}^{\circ} 1$, mai 1988 , p. 122.

23. Dans L'Obéissance, le cliché revient en force, car, signe de complicité avec l'ordre établi, il a partie liée avec la torture que dénonce le roman. 
autre, comme vous le savez déjà." ( $A P D$, p. 20) En somme, on aime comme on voyage, pour l'éclair de l'instant.

Flore, Laura, Galatée, Pomme, Maude et les autres: la féminité est dans l'excès, dans le débordement. Chaque fois qu'on croit l'avoir saisie, cette femme se trouve déjà ailleurs. On ne peut la décrire, sauf en la disant indescriptible. Chaque femme a son image: petite boule de mercure des thermomètres pour Flore Cocon, casse-tête impossible à assembler pour Laura Laur, porte de secours pour Pomme Douly, bref autant de figures de la versatilité et de la fuite. De son amie-amoureuse dépitée, Sylvie Nord, Gala reçoit un cube scellé dont l'intérieur est hérissé de clous; qui tâche de s'emparer de la "bille noire insondable " $(P G$, p. 187) qu'il renferme se déchire la chair sans pouvoir atteindre la bille. La femme jacobienne est une interrogation qui a revêtu un instant la forme humaine, et qui refuse toute clôture, toute réponse définitive ${ }^{24}$.

La seule attitude possible pour nous qui lisons est celle qu'adoptent, bien à contrecour, les amants, les amoureuses qui tiennent à revoir cette femme: ne pas s'étonner de ses intermittences, ne jamais chercher à la retenir. Elle nous réapprend cette vérité essentielle: qui perd gagne. Elle joue, elle se joue, elle se joue de nous. Elle ne vit, en somme, que dans la plus extrême désobéissance. Dans la vie comme en amour, son credo est simple et radical : "[I]l faut y mettre le désordre, tout le désordre possible." $(S$, p. 43)

24. Elle résiste aussi, bien entendu, au métalangage critique qui prétend la cerner, d'où la forme déliée, quelque peu serpentine du présent article. 